\title{
The vigilance components of begging and sibling competition
}

\author{
Amélie N. Dreiss, Marco Calcagno, Valentijn van den Brink, Aurélie Laurent, Bettina Almasi, \\ Lukas Jenni and Alexandre Roulin
}

A. N. Dreiss (amelie.dreiss@unil.ch), M. Calcagno, V. van den Brink and A. Roulin, Dept of Ecology and Evolution, Univ. of Lausanne, CH-1015 Lausanne, Switzerland. - A. Laurent, Univ. of Burgundy, Dijon, France. - B. Almasi and L. Jenni, Swiss Ornithological Inst., CH-6204 Sempach, Switzerland.

\begin{abstract}
The conflict between siblings over how parental resources are divided has promoted the evolution of specific behaviour to outcompete each other. Young animals look out for parents' arrival in order to start begging as quickly as possible, since a rapid begging reaction increases the likelihood of being fed before nestmates. If the young can physically intercept the parents, selection might be operating on the offspring ability to monitor parent arrival (vigilance towards parents) and any sudden modifications in siblings' behaviour (vigilance towards siblings). To investigate the adaptive value of nestling vigilance in the context of family interactions, we recorded which direction barn owl Tyto alba siblings were facing in 89 two-chick broods before the first parental feeding visit of the night. Nestlings were more vigilant towards nest entrance than expected by chance suggesting that vigilance towards parents is an important component of sibling competition. When positioned near the nest-box entrance where parents predictably deliver food, the younger individual (i.e. junior) looked more towards the entrance than its older sibling. Thus, when the likelihood of obtaining a food item is relatively high, juniors are more vigilant than seniors to detect the incoming parent. When positioned at the back of the nest, the senior looked relatively more frequently towards its sibling than the junior did in the same situation. This suggests that when the likelihood of obtaining a food item is relatively low, seniors are more vigilant than juniors to observe their sibling. Because vigilance was not related to hunger level and prey obtaining, we propose the hypothesis that vigilance towards parents and siblings only indirectly influences the outcome of sibling competition.
\end{abstract}

In animals, vigilance has been studied mainly in the context of predator-prey relationships where animals frequently interrupt feeding to look up and visually scan the environment to detect any predator in their surroundings (Bednekoff and Lima 2002, Beauchamp 2003). Individuals can be vigilant not only towards potential predators but also towards conspecifics to avoid negative social interactions such as food theft (Knight and Knight 1986, Jones 1998). This social component of vigilance, so-called 'within-group vigilance' (Treves 2000), is modulated in relation to group dynamics (group size, spatial position of the individuals within the group; Fernandez-Juricic et al. 2004), individual state (size, age, sex and reproductive status; Lung and Childress 2007) and the environment (predation risk and food resources; Roth et al. 2006). For instance, in the eastern grey kangaroo Macropus giganteus, when group size increased, females located at the group periphery decreased the time spent in anti-predator vigilance in favour to withingroup vigilance (Favreau et al. 2010).

An interesting but neglected case of foraging behaviour that can necessitate a high level of vigilance is during sibling contests over parental resources. Several studies showed that the altricial young that starts to beg for food before its siblings is more likely to be fed first by its parent (Teather 1992, Dearborn 1998, Hofstetter and Ritchison 1998,
Lichtenstein and Sealy 1998, Roulin 2001c). Young animals therefore have to be vigilant to detect when parents are back at the nest with food, which requires an ability to distinguish noise produced by an arriving parent from irrelevant stimuli such as the noise made by wind. This is not trivial as nestlings sometimes beg in the absence of parents because they respond to cues that are wrongly interpreted as an arriving parent (Budden and Wright 2001, Leonard and Horn 2001, Dor et al. 2007, but see Roulin 2002 for another adaptive role of this behaviour). Selective attention to beg as quickly as possible once a parent is detected is probably costly, as it requires time and extra brain activity and it prevents young to rest and sleep. Indeed, brain cannot sustain vigilance, such as detection of cryptic targets during long periods of time (e.g. in humans, Nuechterlein et al. 1983, Parasuraman and Mouloua 1987). For this reason, a high level of vigilance can be considered as a costly component of begging that reinforces its honesty as a signal of need (Roulin 2001b).

Usually, researchers interested in parent-offspring interactions only consider the behaviour of young animals in the presence of parents, disregarding processes taking place in their absence (but see for example McRae et al. 1993). This is unfortunate because it is sometimes when parents are foraging away from the nest that priority access to food resources is established among siblings, either by 
communication between siblings (Roulin 2002), aggressive behaviour (Drummond 2006) or jostling for the best position in the nest where parents will deliver food (Kölliker et al. 1998). Furthermore, in parent absence siblings may observe each other to monitor how they will behave upon parent's arrival and outcompete their broodmate by being more vigilant in watching out when parents are back with food, thus intercepting more quickly food items. Siblings may hence increase the likelihood of obtaining a larger than equal share of the impending resources by watching their parents arrival (vigilance towards parents or 'the vigilance component of begging'), but also by observing their siblings to see where they are located and how they react once a parent is detected, a process that we refer to as the 'vigilance towards siblings' or 'the vigilance component of sibling competition'. To the best of our knowledge, to date there is no study that considered the latter component of vigilance.

In the present paper, our aim is to investigate whether vigilance towards parents and siblings are important factors for the resolution of sibling competition in the barn owl Tyto alba. For this aim, we first investigated whether nestlings were more vigilant than expected by chance. We then assessed whether vigilance towards parents and siblings was related to dominance hierarchy, food need and stress, important determinants of resource holding potential and motivation to compete during sibling competition (Roulin 2004a, Ruppli et al. 2012). Finally, to investigate the adaptive value of sibling vigilance, we analysed whether it was related to sibling negotiation and ultimately to prey obtaining. In this species, siblings vocally communicate to each other their hunger level in the absence of parents (Roulin 2002) and this communication, so-called 'sibling negotiation', influences which nestling obtains the prey at parent return (Dreiss et al. 2010). Negotiation calls were defined as calls produced in parent absence, as opposed to begging calls produced in the presence of parents.

We analysed nestling behaviour before the first parental feeding visit of the night in 89 two-chick broods that were video-recorded. We monitored the direction of where these two individuals were looking in relation to the nestbox entrance where parents predictably deliver food and in relation to their sibling. An individual was considered vigilant towards nest entrance (or sibling) when he had the nest entrance (or its sibling) in its visual field. Although barn owl nestlings interact at night, their low-light vision probably allows them to see their surroundings (Schaeffel and Wagner 1996), even in a closed nest where moonlight and candlelight can enter through the entrance. Moreover, when a barn owl is facing an object, it also maximizes its capacity to obtain spatial information from any sound coming from this object, probably due to owl facial ruff (Knudsen and Konishi 1979, Hausmann et al. 2009). In the barn owl, there is a pronounced age hierarchy resulting from hatching asynchrony, the process by which eggs hatch sequentially in time every 2 to $3 \mathrm{~d}$ on average. We thus compared vigilance in relation to age hierarchy, one of the individuals being older (i.e. the senior) than the other (i.e. the junior) and in relation to the position of the two individuals in the nest-box (i.e. close to the nest-box entrance or at the back of the nest), as position determines whether nestlings could be vigilant towards sibling and parents simultaneously. For a number of nests, we manipulated nestling hunger level by experimentally food-depriving or food-satiating individuals and in some other nests we experimentally administrated corticosterone, the hormone that regulates stress. Corticosterone implantation mimics a short-term stress, which could be for instance due to food shortage (Kitaysky et al. 1999). We could thus investigate whether the vigilance towards nest entrance and sibling was related to individual need. Nestlings might be expected to be more vigilant towards parents and competitors when needy, i.e. after food depletion or corticosterone-mediated stress, as a mean to increase chance of obtaining the prey. Conversely, stressful condition might prevent nestlings to invest additional effort in vigilance competition, if vigilance is costly.

\section{Material and methods}

\section{Study organism}

The barn owl is a nocturnal predator that hunts in the open fields. Parents bring a single prey item, usually a vole, per feeding visit occurring on average every $45-60 \mathrm{~min}$. In our study area located in western Switzerland, owls breed in nest-boxes $(1.0 \times 0.6 \times 0.5 \mathrm{~m})$ fastened to barn walls. The 2 to 11 eggs are laid from February to August and since the mother starts incubation just after having laid the first egg, there is a pronounced within-brood age hierarchy with the first-hatched nestling being up to three weeks older than its younger sibling. Compared to senior nestlings juniors invest more effort in vocalization behaviour both in the absence of parents (so-called negotiation) and in their presence (i.e. begging; Roulin 2004a), but they do not differ in the proportion of time they are closer to the nest-box entrance (Dreiss et al. 2010). In two-chick broods an individual has a higher probability of obtaining a prey item when begging for food from parents at a higher rate than its sibling and also if it had produced longer negotiation calls before the arrival of the feeder (Dreiss et al. 2010). Further analyses by Dreiss et al. (2010) showed that being positioned close to the entrance in the absence of parents seems to induce its sibling in the back to negotiate at a lower level which in turn motivates the individual that is close to the entrance to beg at a higher level once a parent is back with food. Thus, being positioned at the front of the nest indirectly increases the likelihood that a parent feeds this individual in priority.

\section{Video recordings}

The study was performed between 1997 and 2005. The present study is based on the same video recordings as the ones used in previous studies for which the aim was to investigate the role of vocalizing on prey obtaining (Dreiss et al. 2010, Ruppli et al. 2012). To create two-chick broods, we temporarily removed all but two randomly chosen nestlings from the nests between 21:30 and 24:00 h. Study broods originally contained two (five broods) to nine nestlings (mean \pm SE: $4.7 \pm 0.2$ ). All nestlings were handled for body mass measurement before experiment at 21:30 h. Using an infrared sensitive camera and a microphone we recorded the 
two individuals until midnight when the removed nestlings were put back in their nest. On video footage we did not notice any sign of distress to either the nestlings or adults. We ringed the two siblings each on a different leg to recognize them and identified which of them obtained the first prey item delivered of the night. Camera and microphone were placed in an upper corner of the nest-box. Because nestlings move within their nest, it is not possible to fix camera and microphone at a fixed distance to nestlings. In five videos out of 89 , we could not observe which nestling obtained the prey, and in four videos, one of the two nestlings was not visible. We measured the length of the flattened wing from the bird's wrist to the tip of the longest primary to estimate age (Roulin 2004b). The oldest nestling, referred to as 'senior', was on average $35.6 \mathrm{~d}$ old (range $=20-54$ ) and the 'junior' sibling on average $30.8 \mathrm{~d}$ (range $=17-52)$. The age difference between the two siblings was on average $4.9 \mathrm{~d}$ $($ range $=1-21)$.

\section{Nestling treatments}

In order to test condition-dependence of vigilance, we analysed two-chick broods for which we manipulated nestling hunger level or circulating corticosterone levels. These two components of condition have already been shown to alter sib-sib communication in the barn owl (Roulin 2002, Ruppli et al. 2012). We recorded nestling behaviour in twochick broods in five years (1997, 2000, 2001, 2004 and 2005; Table 1). In 2000 and 2001 we recorded 22 and 8 two-chick broods, respectively, in 21 and 7 different nests, for which nestlings were not treated in any specific way in contrast to the other years. In 1997, hunger level was manipulated in 12 nests (Roulin et al. 2000). We obtained information on the

Table 1. Sample size of the analysis of vigilance and number of negotiation calls in two-chick barn owl broods. J stands for junior and $\mathrm{S}$ for senior sibling.

\begin{tabular}{|c|c|c|c|}
\hline Treatments & $\begin{array}{c}\text { No. of } \\
\text { studied } \\
\text { videos for } \\
\text { vigilance }\end{array}$ & $\begin{array}{c}\text { No. of } \\
\text { studied } \\
\text { videos for } \\
\text { negotiation }\end{array}$ & $\begin{array}{l}\text { Year of } \\
\text { recording }\end{array}$ \\
\hline No treatment & 22 & . & 2000 \\
\hline No treatment & 8 & . & 2001 \\
\hline J and S food-deprived & 7 & 7 & 1997 \\
\hline $\begin{array}{l}\text { S food-deprived, } \\
\text { J food-satiated }\end{array}$ & 8 & 7 & 1997 \\
\hline $\begin{array}{l}\text { J food-deprived, } \\
\text { S food-satiated }\end{array}$ & 9 & 9 & 1997 \\
\hline $\begin{array}{l}\text { J cort-implanted, } \\
\text { S placebo }\end{array}$ & 8 & . & 2004 \\
\hline $\begin{array}{l}\text { J cort-implanted, } \\
\text { S placebo }\end{array}$ & 8 & . & 2005 \\
\hline $\begin{array}{l}\text { S cort-implanted, } \\
\text { J placebo }\end{array}$ & 11 & . & 2004 \\
\hline $\begin{array}{l}\text { S cort-implanted, } \\
\text { J placebo }\end{array}$ & 8 & . & 2005 \\
\hline
\end{tabular}

No treatment indicates that nestlings were not manipulated. Fooddeprived individuals were prevented to consume food during the preceding $12 \mathrm{~h}$, whereas food was offered ad libitum during the last $12 \mathrm{~h}$ in the so-called 'food-satiated' individuals. 'Cort-implanted' indicates that nestlings were implanted with a corticosteronereleasing pellet, whereas 'placebo' indicates that nestlings were implanted with a similar pellet but that did not contain corticosterone. The senior individual was older than its junior sibling. two vigilance components in seven video recordings where both the junior and senior siblings were food-deprived, eight video recordings where the senior was food-deprived and the junior food-satiated, and nine video recordings where the junior was food-deprived and the senior food-satiated. During the daylight hours from 09:00 to 21:30 we separated the two siblings and kept them in a ventilated box with either three dead mice to food-satiate them or without prey to food-deprive them. At 21:30 we put the two individuals back in their nest-box to record their behaviour. The same two individuals were recorded on three successive nights, one night only the senior was food-satiated, another night only the junior was food-satiated and on the third night the two siblings were food-deprived. The order of the manipulations was randomized across the three nights (for further details see Roulin et al. 2000). This experiment had no detrimental effect on the nestlings (see ethical note in Roulin and Bersier 2007).

In 2004 and 2005 our aim was to study the effect of corticosterone (the main hormone in birds that mediates physiological and behavioural responses to stressors; Veiga et al. 1978) on barn owl nestling behaviour (Ruppli et al. 2012). In two-chick broods one of the individuals was treated with corticosterone by implanting a corticosterone-releasing pellet under the skin and its sibling received a similar pellet that did not contain corticosterone (placebo treatment). We were able to monitor the vigilance towards nest entrance and sibling in 19 two-chick broods where the senior had been treated with corticosterone (11 in 2004 and 8 in 2005), and in 16 two-chick broods where the junior had been treated with corticosterone (8 in each year). As shown in Ruppli et al. (2012) at the time of implantation, corticosterone-implanted individuals did not differ from placebo-individuals in terms of age (2004: $30.0 \pm 0.7 \mathrm{~d}$ vs $31.0 \pm 0.9 \mathrm{~d}$; 2005 : $31.0 \pm 0.9 \mathrm{~d}$ vs $30.0 \pm 1.4 \mathrm{~d}$ ) or body mass. This manipulation increased circulating corticosterone within a physiological range for about two days in corticosterone-implanted individuals compared to placebo individuals (Almasi et al. 2009, Müller et al. 2009). This experiment affected growth (Almasi et al. 2012) and vocalisation behaviour at the time of the video-recording (Ruppli et al. 2012). See Müller et al. (2009) and Ruppli et al. (2012) for further details on the methods. The corticosterone treatment was approved by the Swiss committee for animal research (animal experiment permit no. 1736 from the Veterinarian Office of the 'canton de Vaud').

\section{Assessment of vocal negotiation and individual position in nest-box}

We analysed nestlings' behaviour during the 15 min preceding the first parental feeding visit of the night (i.e. parents were hunting and hence not in the nest-box). For the broods that were food-manipulated in 1997, we counted the number of negotiation calls produced each 20 seconds (Table 1). For all video recordings, we recorded the spatial location in the nest-box of the two owlets every $20 \mathrm{~s}$ during the same $15 \mathrm{~min}$ preceding the first parental visit. Score 1 was assigned to the individual positioned closer to the nest-box entrance and score -1 to its sibling; when the two nest-mates were located at the same distance to the entrance, we assigned the 
score 0 . Then, for each individual we calculated the mean score (in \%) over the 45 assigned scores, which estimated its mean position in its nest-box relative to the nest entrance over the $15 \mathrm{~min}$. The mean position varied from $-100 \%$ when an individual was always closer to the back of the nestbox than its nestmate to $+100 \%$ when the individual was always closer to the nest-box entrance than its nestmate. A mean position of 0 indicates that the two individuals were on average located at the same mean position to the nest-box entrance.

\section{Assessment of the vigilance towards nest entrance and towards sibling}

The vigilance towards nest entrance was defined as the possibility for a nestling to see the nest entrance. The total field of view for an owl is ca $160^{\circ}$, with about $45^{\circ}$ of binocular vision (Knudsen and Knudsen 1989). Barn owl eye movements are highly limited (with a maximum amplitude of about $2^{\circ}$, Steinbach and Money 1973), meaning that the direction where the animal is looking is given by the direction of the face. We estimated the angle between the direction where an individual was looking and the nest-box entrance. The angle was calculated between the vertical line that connected the bill and the centre of the head (midsagittal plane) and the line that connected the centre of the head and the entrance (Fig. 1). This angle ranged from $0^{\circ}$ when nestlings were facing the opening of the nest-box where parents predictably deliver food to $180^{\circ}$ when they were looking in the opposite direction, which can be frequent (Roulin et al. 2008). Thus, an individual was considered vigilant towards nest entrance (and hence better hear and see an arriving parent) when the angle was smaller than $80^{\circ}\left(160^{\circ} / 2\right)$. This measure allows us to estimate vigilance activity, although vigilance can only be measured by brain activity (as an individual facing an object might not be attentive to it if it is sleepy) and the effectiveness of vigilance can only be studied experimentally by the estimate of reaction time (Jakobsson et al. 1995). The vigilance towards sibling was similarly defined as the possibility for an individual to theoretically see (and hence better hear) its sibling, with the same calculation of angles as for the angle to the nest-box entrance (Fig. 1). This angle ranged from a minimum of $0^{\circ}$ when the focal nestling was facing its sibling up to a maximum of $180^{\circ}$ when it was looking in the opposite direction. Again an individual was considered vigilant towards its sibling when the angle was smaller than $80^{\circ}$. The angle was estimated from sibling's head (Fig. 1), but as barn owl nestlings usually stand vertically with body beneath the head, we would obtain very similar angles if we considered the entire siblings' body. For each vigilance component (towards nest entrance or its sibling), every $20 \mathrm{~s}$ during the 15 min preceding the first parental visit, each nestling was assigned score 1 if it was vigilant and 0 otherwise. The mean vigilance during the $15 \mathrm{~min}$ preceding the first parental visit was thus the proportion of scores that were 1 . Note that both the sibling and the nest-box entrance can be in the same field of view.

\section{Statistical procedure}

All the statistical analyses were performed using the statistical software package SAS 9.1. The mean position and the mean vigilance over the $15 \mathrm{~min}$ of observation were arcsine-root transformed to obtain normally distributed variables. Tests

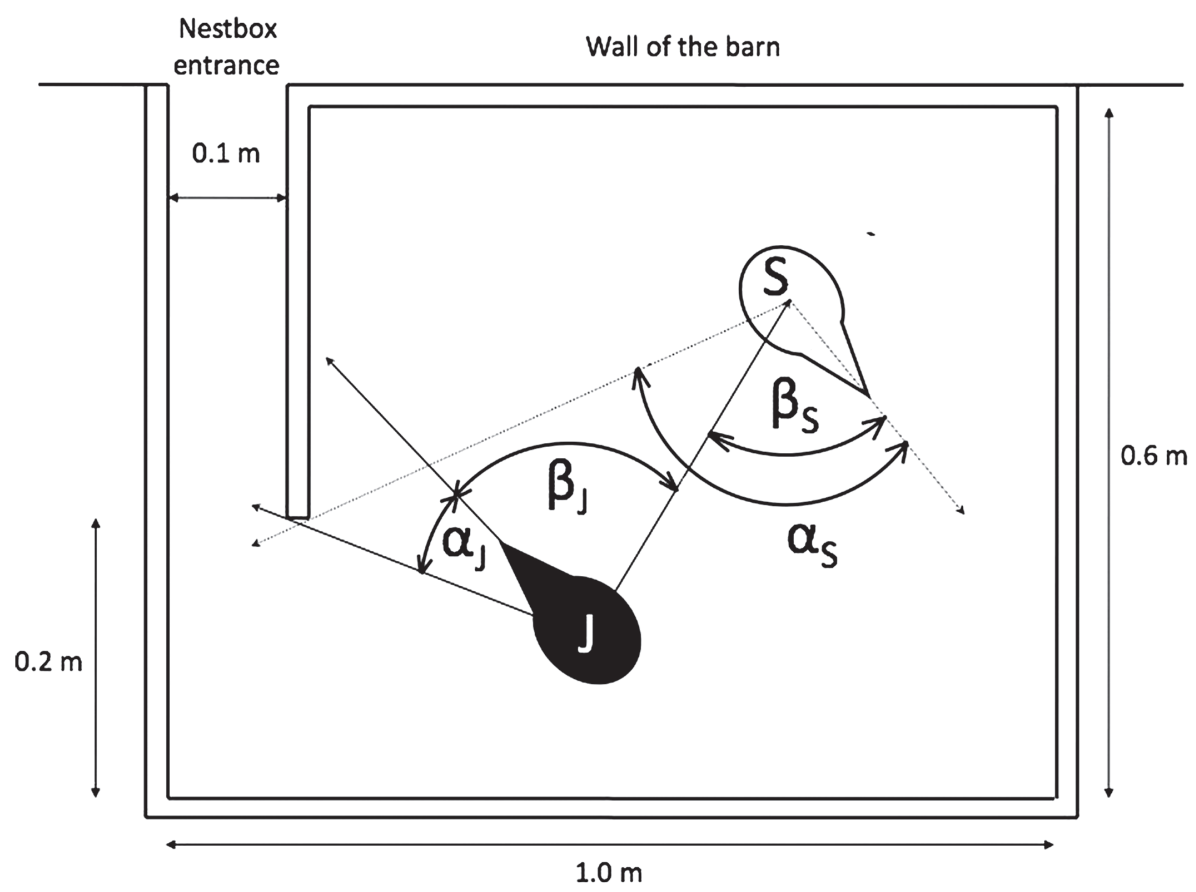

Figure 1. A nest-box with a junior barn owl nestling $(\mathrm{J})$ and its senior sibling $(\mathrm{S})$. The vigilance towards nest entrance is defined by the possibility to see the nest entrance, which is when the angle $(\alpha)$ between the direction where an individual was facing and the nest-box entrance was smaller than $80^{\circ}$. The vigilance towards sibling was defined by the possibility to see its sibling, which is when the angle $(\beta)$ between the direction where an individual was facing and its sibling was smaller than $80^{\circ}$. In this figure the junior is closer to the nest-box entrance than its sibling, and hence it was assigned score 1 and the senior score -1 . 
are two-tailed and p-values smaller than 0.05 are considered significant. Means are quoted $\pm \mathrm{SE}$.

\section{Effect of seniority and position on the vigilance towards nest entrance and sibling}

We performed Wilcoxon signed tests to compare the proportion of time an individual had its sibling (or the nest-box entrance) in its visual field with the random expectation of $44 \%$ (i.e. $\left[160^{\circ} / 360^{\circ}\right] \times 100$ ) for a given object to be by chance in the $160^{\circ}$ visual field of a barn owl. This was done separately for the situation when an individual was behind its sibling and when it was closer to the nest-box entrance than its sibling. Eight signed tests were therefore performed (vigilance towards nest entrance and sibling for when the focal junior and senior individual was behind and in front of its nestmate). For example, in Fig. 1 the junior individual $J$ has its senior sibling $S$ in its visual field if the angle $B_{j}$ is smaller than $80^{\circ}$; similarly the senior $S$ has its junior sibling $\mathrm{J}$ in its visual field if the angle $\beta_{\mathrm{S}}$ is smaller than $80^{\circ}$. We further analysed whether the within-individual change in vigilance, during the 15 min observation period, was related to the within-individual change in position. Using paired t-tests we thus compared the mean vigilance of each individual when positioned in the front with its average vigilance when positioned in the back. We thus considered only 66 senior and 65 junior individuals for which we had data in these two situations. In preliminary analyses, we established that the results were the same whether we consider all the years or only the years without manipulation. We therefore report the results based on the entire sample.

To investigate the effect of food (food-deprived vs foodsatiated) and corticosterone treatments (corticosteroneimplanted vs placebo) on the two vigilance components during the 15 min preceding the first parental visit, we performed linear mixed models. The dependent terms in two separated models were the proportion of time an individual was vigilant towards nest entrance and the proportion of time it was vigilant towards sibling. The independent variables were treatment (six levels: food-deprived focal individual competing with a food-deprived sibling, food-deprived focal individual competing with a food-satiated sibling, foodsatiated focal individual competing with a food-deprived sibling, non-treated focal individual competing with a nontreated sibling, corticosterone-implanted focal individual competing with placebo sibling and placebo focal individual competing with a corticosterone-implanted sibling), seniority (junior vs senior), absolute age difference between the two siblings and mean position in the nest-box (i.e. the proportion of time an individual was in front (1 to $100 \%$ ) or behind ( -1 to $-100 \%)$ its nestmate). Interactions containing the term absolute age difference were never significant and were not reported for the sake of clarity. Because 11 nests were recorded in more than one night (e.g. nestlings could have experienced different food treatments in different nights) and because per video recording we always recorded two siblings, we introduced the video (i.e. first, second or third night of recording) nested in both brood identity and year as random factors); note that if we replace the term 'video' by 'nestling identity' we obtain qualitatively similar final models. Our data structure does not allow us including nestling identity nested in video as random intercepts. However, if we run a model with video nested in brood nested in year and another model with nestling identity nested in brood nested in year, the random factor video explained more variance than the random factor nestling identity. Therefore, we kept the model with video as random variable for all further analyses. We performed model selection by backward elimination of the non-significant terms $(p>0.05)$ beginning with the highest order interaction terms. Final models contained only significant variables and variables involved in significant interactions. The number of nestlings originally present in the nest (between two and nine) was not significantly related to the two vigilance components, alone or in interaction with other independent variables. Hence, we did not include this variable in the models presented here.

\section{Vigilance and sibling negotiation}

We analysed whether the within-individual fluctuation in vocal negotiation over the $15 \mathrm{~min}$ observation period was related to the within-individual fluctuation in vigilance using the vocal data collected in 1997 . Whether nestlings produced a negotiation call during a given $20 \mathrm{~s}$ period (yes or no) was set as a dependent variable in a generalized mixed model with binomial distribution. As independent terms, we computed the vigilance (yes or no) of focal individual and of its partner individual towards the nest entrance as well as towards its siblings. Sibling position score during the same $20 \mathrm{~s}$ and treatment were included as cofactors. As each nestling was recorded several times during the $15 \mathrm{~min}$ preceding the first parental visit, time was set as covariate and individual identity nested in the night of video recording was set as random factor, as well as brood identity.

\section{Vigilance and prey obtaining}

A generalized mixed model with binomial distribution was performed to investigate whether the probability that the junior nestling received the prey item was related to the two different components of vigilance measured in juniors and seniors and to their mean position in the nest-box (variation between $-100 \%$ to $100 \%$ ) during the 15 min preceding the first parental feeding visit of the night. We had one value per nest, but as some nests were recorded in more than one night (see above), nest identity and year were included in the model as random variables.

\section{Results}

In $30 \%$ of observations, individuals were vigilant towards both their sibling and the nest entrance, while in $18 \%$ they were neither vigilant towards their sibling nor the entrance. In $43 \%$ of observations they were only vigilant towards nest entrance and in $9 \%$ only towards their sibling.

\section{Vigilance towards nest entrance}

Seniors and juniors were more vigilant towards the nest-box entrance than the $44 \%$ random expectation, both when they were closer than their nestmate to the back of their nestbox (proportion of time the nest entrance was in the visual field: seniors: $74 \% \pm 3$, signed test: $S=1087, p<0.0001$, 
$\mathrm{n}=72$; juniors: $69 \% \pm 3, \mathrm{~S}=1166, \mathrm{p}<0.0001, \mathrm{n}=80$ ) and when closer to the nest-box entrance (seniors: $76 \% \pm 3$, $\mathrm{S}=1509, \mathrm{p}<0.0001, \mathrm{n}=81$; juniors: $80 \% \pm 2, \mathrm{~S}=1237$, $\mathrm{p}<0.0001, \mathrm{n}=72$ ).

When analysing between-individuals variation in behaviour by using mean individual values, the mean vigilance towards nest entrance (i.e. the proportion of time nestlings could see the nest-box entrance during the 15 min preceding the first parental feeding) was related to the interaction between seniority and nestling mean position (linear mixed model in Table 2a). The significant interaction is explained by the fact that when juniors were positioned closer to the nest-box entrance than their sibling, they were slightly more vigilant towards the opening than when located further away from the entrance (similar linear mixed model considering only juniors: $\mathrm{F}_{1,77}=4.39, \mathrm{p}=0.039$; Fig. 2a; estimate: $0.16 \pm 0.07$ ), whereas seniors tended to be less vigilant towards the opening when positioned closer than further away from the nest-box entrance than their nestmate, although not significantly so (similar linear mixed model considering only seniors: $\mathrm{F}_{1,81}=2.18, \mathrm{p}=0.14$; Fig. 2a; estimate: $-0.10 \pm 0.07)$. The manipulation of food supply and corticosterone did not affect the vigilance towards nest entrance, neither alone nor in interaction with mean position in the nest-box or seniority (all p-values $>0.54$; Table $2 \mathrm{a}$ ). This result remains unchanged when analysing food-treatment (1997) and corticosterone treatment

Table 2. Effect of treatment, position in the nest and seniority on the vigilance towards nest entrance (i.e. proportion of time a focal nestling could see the entrance of its nest-box) and sibling (i.e. proportion of time a focal nestling could see its nestmate) in twochick barn owl broods ( $\mathrm{n}=89$ video recordings of 147 individuals in 74 nests). The night when two siblings were recorded was nested in the brood they were raised and year and implemented as random factor in linear mixed models. Results of final models are written in bold and non-significant results eliminated from the initial full models in plain.

\begin{tabular}{|c|c|c|c|c|c|c|}
\hline \multirow[b]{2}{*}{ Effect } & \multicolumn{3}{|c|}{$\begin{array}{l}\text { (a) Vigilance towards } \\
\text { nest entrance }\end{array}$} & \multicolumn{3}{|c|}{$\begin{array}{l}\text { (b) Vigilance towards } \\
\text { sibling }\end{array}$} \\
\hline & DF & $\mathrm{F}$ & $\mathrm{p}$ & DF & $\mathrm{F}$ & $\mathrm{p}$ \\
\hline Seniority & 1,83 & 5.04 & 0.03 & 1,83 & 7.54 & 0.007 \\
\hline Treatment & 5,81 & 1.18 & 0.32 & 5,81 & 0.79 & 0.57 \\
\hline Position & 1,83 & 0.40 & 0.53 & 1,83 & 58.46 & $<0.0001$ \\
\hline $\begin{array}{c}\text { Absolute age } \\
\text { difference }\end{array}$ & 1,81 & 0.99 & 0.32 & 1,81 & 0.20 & 0.65 \\
\hline $\begin{array}{l}\text { Seniority } \times \\
\text { Treatment }\end{array}$ & 5,75 & 0.52 & 0.76 & 5,75 & 0.75 & 0.59 \\
\hline $\begin{array}{l}\text { Position } \times \\
\text { Seniority }\end{array}$ & 1,83 & 7.74 & 0.007 & 1,83 & 4.48 & 0.04 \\
\hline $\begin{array}{l}\text { Position } \times \\
\text { Treatment }\end{array}$ & 5,75 & 0.85 & 0.52 & 5,75 & 2.07 & 0.08 \\
\hline
\end{tabular}

Treatment consisted in either the manipulation of food supply, corticosterone levels or absence of any of these treatments. Foodtreatment consisted in food-depriving both siblings during the preceding $12 \mathrm{~h}$ (a normal situation and hence also called absence of any treatment), in food-satiating both individuals (i.e. offered food ad libitum during the last $12 \mathrm{~h}$ ), or in food-depriving one individual and food-satiating the other. In the corticosterone experiment, we implanted one individual with a corticosterone-releasing pellet and the other individual was implanted with a placebo pellet. The term 'position' indicates the proportion of time an individual is closer to the nest-box entrance than its nestmate (variation between 0 and $100 \%$ ) or is closer to the back of the nest-box than its nestmate (variation between 0 and $-100 \%$ ). The term 'seniority' indicates which individual is older (i.e. senior) than the other (i.e. junior).
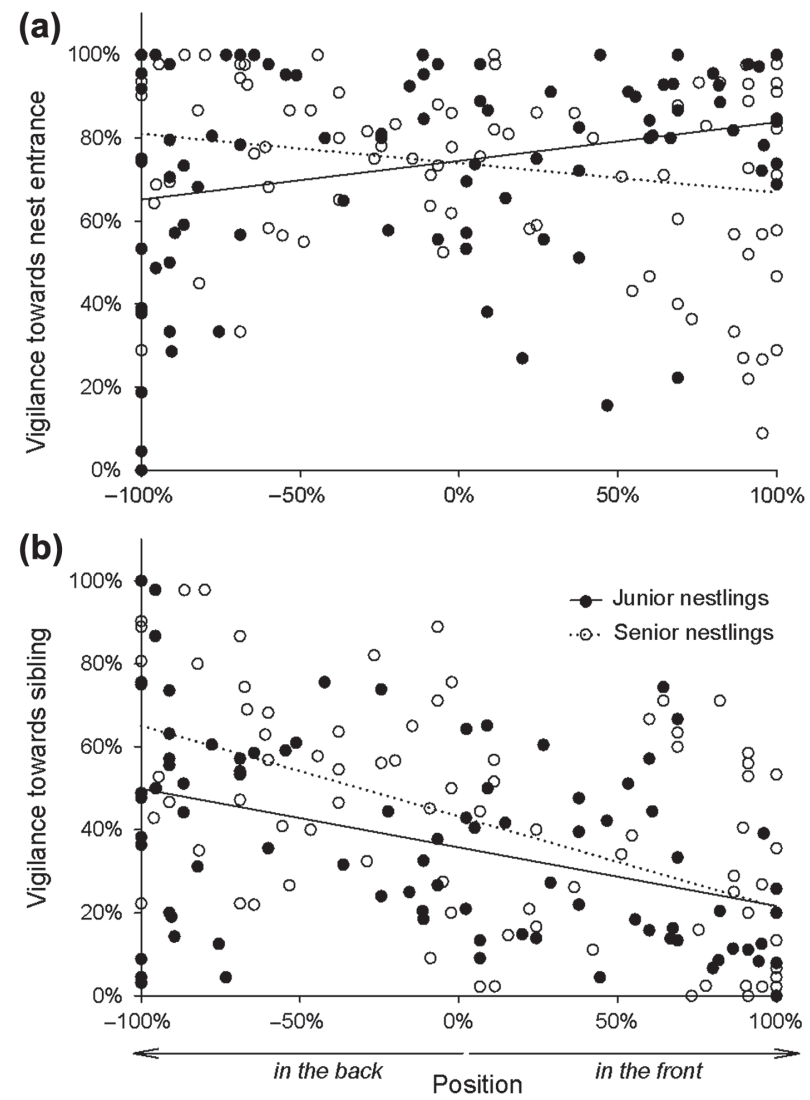

Figure 2. Among-individual variation in the level of vigilance towards nest entrance (a) and the sibling (b), estimated as the proportion of time an individual had the nest-box entrance (a) and its sibling in its visual field (b), according to the position in the nest. Data are from two-chick barn owl broods. Each data point represents a mean individual value. Position in the nest is defined as the proportion of time an individual was closer to the nest entrance than its sibling (variation between $0 \%$ and $100 \%$ ) and closer to the back of the nest-box (variation between $-100 \%$ and $0 \%$ ).

(2004-2005) in two separated similar mixed models (foodtreatment effect: $\mathrm{F}_{1,49}=1.26, \mathrm{p}=0.30$; corticosteronetreatment effect: $\mathrm{F}_{1,30}=2.20, \mathrm{p}=0.15$ ).

These between-individual analyses are confirmed by a within-individual analysis. Indeed, seniors did not differ in their vigilance towards nest entrance according to their position in the nest (paired t-test, comparison of the mean individual vigilance when individual was in the front and in the back: $\mathrm{t}_{65}=0.49, \mathrm{p}=0.62$ ), while juniors were more vigilant towards nest entrance when in the front than in the back of their nest-box (paired t-test, $\mathrm{t}_{64}=2.50, \mathrm{p}=0.015$ ).

\section{Vigilance towards sibling}

When positioned at the back of the nest-box while their sibling was closer to the nest-box entrance, both seniors and juniors were more vigilant towards their sibling than the random expectation of $44 \%$ (proportion of time the nestmate was in the visual field: seniors: $63 \% \pm 4$, signed-test: $\mathrm{S}=777, \mathrm{p}<0.0001, \mathrm{n}=71$; juniors: $53 \% \pm 3, \mathrm{~S}=468$, $\mathrm{p}=0.021, \mathrm{n}=79$ ). By contrast, when positioned closer than their nestmate to the entrance of the nest-box, seniors and juniors were less vigilant towards their sibling than the 
$44 \%$ random expectation (seniors: $25 \% \pm 3, S=1055$, $\mathrm{p}<0.0001, \mathrm{n}=80$; juniors: $25 \% \pm 3, \mathrm{~S}=907, \mathrm{p}<0.0001$, $\mathrm{n}=70$ ). This pattern could have resulted from the high vigilance of nestlings towards nest entrance. Accordingly, we found that within-individuals both senior and junior nestlings were more vigilant towards their sibling when positioned in the back than in the front (paired t-test, comparison of the mean individual vigilance when individual was in front and in the back; seniors: $\mathrm{t}_{65}=7.33, \mathrm{p}<0.0001$; juniors: $\left.\mathrm{t}_{63}=5.72, \mathrm{p}<0.0001\right)$.

The among-individuals analysis using mean individual values showed that seniors were more vigilant towards their sibling compared to juniors (estimate for juniors vs seniors: $-0.078 \pm 0.043$; Table 2b). Both nestlings were more vigilant towards their sibling when positioned at the back than front of their nest-box (linear mixed model, position effect for seniors: $-0.45 \pm 0.07, \mathrm{~F}_{1,79}=43.50, \mathrm{p}<0.001$; juniors: $-0.25 \pm 0.06, F_{1,79}=16.37, p=0.001$; Fig. 2b). As a consequence, the vigilance levels of seniors and juniors towards their sibling were negatively correlated (Pearson's correlation on mean vigilance per individual: $r=-0.32$, $\mathrm{n}=86$ pairs of siblings, $\mathrm{p}=0.003$ ). The manipulation of food supply and corticosterone did not affect the vigilance towards sibling, neither alone nor in interaction with mean position in the nest-box or seniority (all p-values $>0.08$; Table $2 \mathrm{~b}$ ). This result remains unchanged when analysing food-treatment (1997) and corticosterone treatment (2004-2005) in two separated similar mixed models (food-treatment effect: $\mathrm{F}_{1,49}=0.28, \mathrm{p}=0.84$; corticosterone-treatment effect: $\mathrm{F}_{1,30}=2.20, \mathrm{p}=0.15$ ).

\section{Vigilance and sibling negotiation}

Independently of seniority, a nestling was more likely to produce at least one negotiation call during an observation session of $20 \mathrm{~s}$ when it was vigilant towards the nest entrance compared to the situation where it was not vigilant towards the entrance (probability of negotiating: $0.54 \pm 0.01$ vs $0.38 \pm 0.02$; Table 3 ). Conversely, a nestling was more likely to produce at least one negotiation call when its sibling was not vigilant rather than vigilant towards the entrance (probability of negotiating: $0.39 \pm 0.01$ vs $0.53 \pm 0.02$; Table 3 ). The mean vigilance level towards the nest entrance was not correlated between siblings (Pearson's correlation: $r=-0.03$, $\mathrm{n}=86, \mathrm{p}=0.77$ ) and hence these findings suggest that the effects of vigilance towards nest entrance of both siblings were independent. As already shown in previous studies (Roulin et al. 2000), nestlings are more likely to vocally negotiate when hungry compared to satiated and when facing a hungry than satiated sibling (probability of negotiating for fooddeprived individual competing with a food-satiated sibling: $0.77 \pm 0.02$; for food-deprived individual competing with a food-deprived sibling: $0.44 \pm 0.02$; for food-satiated individual competing with a food-deprived sibling: $0.14 \pm 0.01$ ). The probability to negotiate increased with time (i.e. along the 15 min preceding the parental arrival; Table 3).

\section{Vigilance and obtaining prey}

The first prey item delivered of the night by a parent was as often eaten by juniors as seniors (respectively $n=46$ and
Table 3. Within-individual variation in vocal negotiation according to nestling vigilance towards nest entrance and towards sibling barn owls, during the 15 min preceding the first parental feeding visit of the night. Results are from a generalized mixed model with binomial distribution. Sample size is 23 video recordings of 15 min where data could be taken in 22 individuals from 11 nests. Behaviour was recorded every $20 \mathrm{~s}$ and the vigilance components were defined as whether an individual could see the nest entrance or its sibling during the 15 min preceding the first parental feeding visit of the night. Results of final model are written in bold and non-significant results eliminated from the initial full models in plain.

\begin{tabular}{|c|c|c|c|}
\hline Effect & DF & $\mathrm{F}$ & $\mathrm{p}$ \\
\hline Time & 1,1515 & 6.43 & 0.011 \\
\hline $\begin{array}{l}\text { Vigilance towards nest } \\
\text { entrance (EV) }\end{array}$ & 1,1515 & 8.33 & 0.004 \\
\hline $\begin{array}{l}\text { Partner vigilance towards } \\
\text { nest entrance }\end{array}$ & 1,1515 & 13.47 & 0.0003 \\
\hline Vigilance towards sibling (SV) & 1,1480 & 0.65 & 0.42 \\
\hline $\begin{array}{l}\text { Partner vigilance towards } \\
\text { sibling }\end{array}$ & 1,1480 & 0.45 & 0.50 \\
\hline Position & 1,1514 & 3.38 & 0.07 \\
\hline Seniority & 1,1480 & 0.01 & 0.91 \\
\hline Absolute age difference & 1,1480 & 0.05 & 0.83 \\
\hline Treatment & 1,1515 & 23.13 & $<0.0001$ \\
\hline Position $\times$ EV & 1,1477 & 3.34 & 0.07 \\
\hline Position $\times$ Partner EV & 1,1472 & 2.63 & 0.11 \\
\hline Position $\times$ SV & 1,1472 & 0.80 & 0.37 \\
\hline Position $\times$ Partner SV & 1,1472 & 0.02 & 0.88 \\
\hline Seniority $\times E V$ & 1,1472 & 0.89 & 0.35 \\
\hline Seniority $\times$ Partner EV & 1,1478 & 2.49 & 0.12 \\
\hline Seniority $\times$ SV & 1,1477 & 2.64 & 0.11 \\
\hline Seniority $\times$ Partner SV & 1,1496 & 2.07 & 0.15 \\
\hline
\end{tabular}

38, binomial test: $\mathrm{p}=0.45)$. The probability that nestlings obtained the first prey item of the night was higher when they were positioned near the nest-box entrance than their nestmate (generalized mixed model, Table 4; estimate for position: $1.22 \pm 0.51$; probability of prey obtaining when nearer to entrance than nestmate: $0.61 \pm 0.08$; when farther to entrance than nestmate: $0.49 \pm 0.08$ ), but it was not related to the vigilance towards nest entrance and towards sibling (Table 4).

\section{Discussion}

The present study adds new information on behavioural interactions taking place in the absence of parents between barn owl siblings. Our results show that nestlings were more often facing the nest-box entrance where their parents are bringing food than facing their sibling with whom they are competing to monopolize parental food resources (ca $80 \%$ vs $40-45 \%$ of the time). In only $9 \%$ of observations the nestlings were turned towards their siblings so that they could no more see the nest entrance, while in $43 \%$ of observations they could only see the nest entrance but not their sibling. As nestlings' eye position was significantly different from the random expectation, we could interpret this eye positioning as an active visual vigilance towards nest entrance. This non-random position suggests that being vigilant towards parent arrival has a function. In line with this interpretation, we found that the degree of vigilance was associated with vocalization behaviour: a given individual was more likely 
Table 4. Probability that in two-chick barn owl broods the younger individual (i.e. junior) received the first prey item delivered of the night by a parent according to the different components of vigilance. We report results of a generalized mixed model with binomial distribution and as random factors brood nested in year. Sample size is 85 video recordings taken in 71 nests. The vigilance components were defined as the proportion of time an individual could see the nest entrance or its sibling during the $15 \mathrm{~min}$ preceding the first parental feeding visit of the night. Results of final model are written in bold and non-significant results eliminated from the initial full models in plain.

\begin{tabular}{|c|c|c|c|}
\hline Effect & DF & $\mathrm{F}$ & $\mathrm{p}$ \\
\hline $\begin{array}{l}\text { Junior vigilance towards nest } \\
\text { entrance (junior EV) }\end{array}$ & 1,10 & 0.86 & 0.37 \\
\hline $\begin{array}{l}\text { Senior vigilance towards nest } \\
\text { entrance (senior EV) }\end{array}$ & 1,11 & 1.88 & 0.19 \\
\hline $\begin{array}{l}\text { Junior vigilance towards sibling } \\
\text { (junior SV) }\end{array}$ & 1,12 & 1.00 & 0.34 \\
\hline $\begin{array}{l}\text { Senior vigilance towards sibling } \\
\quad \text { (senior SV) }\end{array}$ & 1,9 & 0.11 & 0.75 \\
\hline Junior position & 1,13 & 5.82 & 0.031 \\
\hline Absolute age difference & 1,13 & 2.53 & 0.14 \\
\hline Junior EV $\times$ Position & 1,7 & 0.73 & 0.22 \\
\hline Senior EV $\times$ Position & 1,5 & 0.03 & 0.87 \\
\hline Junior SV × Position & 1,6 & 0.12 & 0.74 \\
\hline Senior SV $\times$ Position & 1,8 & 1.29 & 0.29 \\
\hline
\end{tabular}

to vocally negotiate with its sibling if vigilant towards the nest-box entrance and if its sibling was not vigilant towards the entrance. The vigilance towards nest entrance is therefore associated with intensity of sib-sib communication, which then in part determines the level with which each competitor begs for food towards parents, the key factor that determines which individual has priority access to food resources (Dreiss et al. 2010). However, in two-chick broods, the interindividual variation in vigilance towards nest entrance did not predict which of the two individuals obtained the next food item delivered by a parent. This suggests that sibling vigilance is not the crucial factor but probably only one parameter out of the multiple elements that indirectly favours nestling food obtaining. In the following, we discuss the determinism of the vigilance towards the nest entrance and towards a sibling, as well as the potential adaptive value of these behaviours.

\section{Vigilance towards nest entrance}

The tendency of an individual to be vigilant towards the nest entrance was not related to hunger level or the manipulation of corticosterone. This suggests that being vigilant is not or only weakly condition-dependent, at least to a lower magnitude than vocal behaviour (Roulin et al. 2000, 2009) and jostling for position (Ruppli et al. 2012). In contrast, the two vigilance components were sensitive to both position in the nest and seniority. Juniors, but not seniors, increased the proportion of time they were vigilant towards the nest entrance when located close compared to away from the entrance. Thus, being vigilant towards the nest entrance where parents deliver food seems to be particularly important in juniors that can be easily physically outcompeted by their older, stronger senior siblings. It indicates that when the likelihood of obtaining the next delivered prey item is relatively high, juniors disproportionally invest in competitive behaviours compared to their older senior sibling, i.e. they call more intensely (Roulin 2004a) and produce longer calls (Roulin et al. 2009), behaviour that increases the likelihood of being fed (Roulin 2001a), and they are also more vigilant by watching out for the parents' return (present study). Therefore, although being vigilant towards the nest entrance is unlikely to causally determine which nestling will be fed before its nestmate, this behaviour is associated with a suite of behaviours that are important to succeed in sibling competition. Moreover, we found that shortterm variation in vocal investment in sibling negotiation was associated with short-term variation in being vigilant towards the nest entrance. Independently of seniority, a nestling was more likely to vocalize in the absence of parents when it was vigilant towards the nest entrance (compared to a situation where this individual was not vigilant towards the entrance) and, more importantly, when its nestmate was not vigilant towards the entrance (compared to a situation where its nestmate was vigilant towards the entrance). The latter result is consistent with the sibling negotiation hypothesis postulating that a nestling will retreat from a contest when its sibling is motivated to compete over the next delivered food item.

Our results therefore showed that even in a nocturnal species, nestlings are vigilant towards nest entrance where their parents arrive with food. This indicates that offspring vigilance is probably developed in other systems. We expect that offspring vigilance towards parents would be particularly high when parents arrive at unpredictable time (such as in the barn owl), at variable place (e.g. in precocial species) and when competition between siblings for food is intense.

\section{Vigilance towards sibling}

When positioned in the back of the nest, senior nestlings were more attentive to their sibling than juniors, and they stayed vigilant towards the nest entrance. When positioned in the back, the chance of obtaining a prey item is lower. This might indicate that seniors remained vigilant to sibling movement and parent arrival even when their chance of obtaining the prey is relatively low. In contrast, when placed at the back of the nest juniors may give up from the contest and hence rest instead of having an eye on siblings. It is plausible that younger, outcompeted individuals need more time to sleep and rest in order to invest resources in competitive interactions only when their outcomes are in their favour.

Barn owl nestlings did not actively keep an eye on their sibling as soon as they were positioned in the front, near the nest entrance. The probability that nestlings' eye position was directed towards their sibling was indeed not significantly different from the random expectation, as long as their sibling was not in the same direction than the nest-box entrance. Moreover, vocal negotiation, which is directed to siblings rather than parents (Roulin et al. 2000), was not related to vigilance towards sibling but towards the nest entrance. Even when positioned in the back of the nest, the vigilance towards nest entrance was higher than towards sibling $(74 \pm 3 \%$ vs $63 \pm 3 \%$ for seniors and $69 \pm 4 \%$ vs $53 \pm 3 \%$ for juniors). This suggests that vigilance towards siblings is not a significant component of sibling competition, even in this species where sib-sib communication is essential for food allocation (Roulin 2002, Dreiss et al. 2010), where nestlings 
appear to assess each other's position and precise vocalisation feature (Dreiss et al. 2010) and where they happen to feed each other (Marti 1989, Roulin et al. 2012). This suggests that in the barn owl sibling communication is essentially vocal, as shown in a previous study (Dreiss et al. 2010) and that the visual communication has a limited role in this system. This is not so surprising given that barn owls are active mainly at night when it can be difficult to accurately see nestmates. We thus expect that vigilance towards siblings would be higher in diurnal species and when competition between siblings leads to aggressive behaviour. Subordinate individual would thus gain by being vigilant towards an aggressive nestmate. Moreover, in precocial species such as Adélie penguins Pygoscelis adeliae, individuals have no nest where parents distribute food and the offspring run after adults to beg for food (Boersma and Davis 1997). In such a system, siblings may increase the likelihood of obtaining food by watching where their siblings go, as they may give them cue of the parent localisation. The vigilance towards siblings could also be different when brood size is smaller because individuals have fewer siblings to watch. Our results might reflect the fact that barn owls are usually raised in larger than two-chick broods and visually watching all surrounding nestmates may be difficult. Our results on two-chick broods were not influenced by the size of the original brood nestlings were raised in. However, the investment in vigilance towards siblings and parents and its correlation with negotiation and food obtaining may change in larger than two-chick broods. Nevertheless, our study is a further step into understanding the complexity of family interactions.

Acknowledgements - AND and MC contributed equally to this work. We thank Wolf Harmening for his advices. The study was financed by the Swiss National Science Foundation (SNF) to AR (grant no. 31003A_120517).

\section{References}

Almasi, B., Roulin, A., Jenni-Eiermann, S., Breuner, C. W. and Jenni, L. 2009. Regulation of free corticosterone and CBG capacity under different environmental conditions in altricial nestlings. - Gen. Compar. Endocrinol. 164: 117-124.

Almasi, B., Roulin, A., Korner-Nievergelt, F., Jenni-Eiermann, S. and Jenni, L. 2012. Coloration signals the ability to cope with elevated stress hormones: effects of corticosterone on growth of barn owls are associated with melanism. - J. Evol. Biol. 25: 1189-1199.

Beauchamp, G. 2003. Group-size effects on vigilance: a search for mechanisms. - Behav. Processes 63: 111-121.

Bednekoff, P. A. and Lima, S. L. 2002. Why are scanning patterns so variable? An overlooked question in the study of antipredator vigilance. - J. Avian Biol. 33: 143-149.

Boersma, P. D. and Davis, L. S. 1997. Feeding chases and food allocation in Adélie penguins, Pygoscelis adeliae. - Anim. Behav. 54: 1047-1052.

Budden, A. E. and Wright, J. 2001. Falling on deaf ears: the adaptive significance of begging in the absence of a parent. - Behav. Ecol. Sociobiol. 49: 474-481.

Dearborn, D. C. 1998. Begging behavior and food acquisition by brown-headed cowbird nestlings. - Behav. Ecol. Sociobiol. 43: 259-270.
Dor, R., Kedar, H., Winkler, D. W. and Lotem, A. 2007. Begging in the absence of parents: a "quick on the trigger" strategy to minimize costly misses. - Behav. Ecol. 18: 97-102.

Dreiss, A. N., Lahlah, N. and Roulin, A. 2010. How siblings adjust sib-sib communication and begging signals to each other. - Anim. Behav. 80: 1049-1055.

Drummond, H. 2006. Dominance in vertebrate broods and litters. - Q. Rev. Biol. 81: 3-32.

Favreau, F. R., Goldizen, A. W. and Pays, O. 2010. Interactions among social monitoring, anti-predator vigilance and group size in eastern grey kangaroos. - Proc. R. Soc. B 277: 2089-2095.

Fernandez-Juricic, E., Siller, S. and Kacelnik, A. 2004. Flock density, social foraging, and scanning: an experiment with starlings. - Behav. Ecol. 15: 371-379.

Hausmann, L., von Campenhausen, M., Eendler, F., Singerheiser, M. and Wagner, H. 2009. Improvements of sound localization abilities by the facial ruff of the barn owl (Tyto alba) as demonstrated by virtual ruff removal. - PloS One 4: e7721.

Hofstetter, S. H. and Ritchison, G. 1998. The begging behavior of nestling eastern screech owls. - Wilson Bull. 110: 86-92.

Jakobsson, S., Brick, O. and Kullberg, C. 1995. Escalated fighting behavior incurs increased predation risk. - Anim. Behav. 49: 235-239.

Jones, M. E. 1998. The function of vigilance in sympatric marsupial carnivores: the eastern quoll and the Tasmanian devil. - Anim. Behav. 56: 1279-1284.

Kitaysky, A. S., Piatt, J. F., Wingfield, J. C. and Romano, M. 1999. The adrenocortical stress-response of black-legged kittiwake chicks in relation to dietary restrictions. - J. Compar. Physiol. B 169: 303-310.

Knight, S. K. and Knight, R. L. 1986. Vigilance patterns of bald eagles feeding in groups. - Auk 103: 263-272.

Knudsen, E. I. and Konishi, M. 1979. Mechanisms of sound localization in the barn owl (Tyto alba). - J. Compar. Physiol. 133: $13-21$.

Knudsen, E. I. and Knudsen, P. F. 1989. Vision calibrates sound localization in developing barn owls. - J. Neurosci. 9: 3306-3313.

Kölliker, M., Richner, H., Werner, I. and Heeb, P. 1998. Begging signals and biparental care: nestling choice between parental feeding locations. - Anim. Behav. 55: 215-222.

Leonard, M. L. and Horn, A. G. 2001. Begging calls and parental feeding decisions in tree swallows (Tachycineta bicolor). - Behav. Ecol. Sociobiol. 49: 170-175.

Lichtenstein, G. and Sealy, S. G. 1998. Nestling competition, rather than supernormal stimulus, explains the success of parasitic brown-headed cowbird chicks in yellow warbler nests. - Proc. R. Soc. B 265: 249-254.

Lung, M. A. and Childress, M. J. 2007. The influence of conspecifics and predation risk on the vigilance of elk (Cervus elaphus) in Yellowstone National Park. - Behav. Ecol. 18: 12-20.

Marti, C. D. 1989. Food sharing by sibling common barn owls. - Wilson Bull. 101: 132-134.

McRae, S. B., Weatherhead, P. J. and Montgomerie, R. 1993. American robin nestlings compete by jockeying for position. - Behav. Ecol. Sociobiol. 33: 101-106.

Müller, C., Almasi, B., Roulin, A., Breuner, C. W., Jenni-Eiermann, S. and Jenni, L. 2009. Effects of corticosterone pellets on baseline and stress-induced corticosterone and corticosteroid-binding-globulin. - Gen. Compar. Endocrinol. 160: 59-66.

Nuechterlein, K. H., Parasuraman, R. and Jiang, Q. 1983. Visual sustained attention: image degradation produces rapid sensitivity decrement over time. - Science 220: 327-329.

Parasuraman, R. and Mouloua, M. 1987. Interaction of signal discriminability and task type in vigilance decrement. - Perception Psychophysics 41: 17-22. 
Roth, T. C., Lima, S. L. and Vetter, W. E. 2006. Determinants of predation risk in small wintering birds: the hawk's perspective. - Behav. Ecol. Sociobiol. 60: 195-204.

Roulin, A. 2001a. Food supply differentially affects sibling negotiation and competition in the barn owl (Tyto alba). - Behav. Ecol. Sociobiol. 49: 514-519.

Roulin, A. 2001b. On the cost of begging vocalization: implications of vigilance. - Behav. Ecol. 12: 506-511.

Roulin, A. 2001c. Screaming as a strategy to reduce the predation risk incurred by begging? - Behaviour 138: 615-627.

Roulin, A. 2002. The sibling negotiation hypothesis. - In: Wright, J. and Leonard, M. L. (eds), The evolution of begging: competition, cooperation and communication. Kluwer Academic Press, pp. 107-127.

Roulin, A. 2004a. Effects of hatching asynchrony on sibling negotiation, begging, jostling for position and within-brood food allocation in the barn owl, Tyto alba. - Evol. Ecol. Res. 6: 1083-1098.

Roulin, A. 2004b. The function of food stores in bird nests: observations and experiments in the barn owl Tyto alba. - Ardea 92: 69-78.

Roulin, A. and Bersier, L. F. 2007. Nestling barn owls beg more intensely in the presence of their mother than in the presence of their father. - Anim. Behav. 74: 1099-1106.

Roulin, A., Kolliker, M. and Richner, H. 2000. Barn owl (Tyto alba) siblings vocally negotiate resources. - Proc. R. Soc. B 267: 459-463.
Roulin, A., Colliard, C., Russier, F., Fleury, M. and Grandjean, V. 2008. Sib-sib communication and the risk of prey theft in the barn owl Tyto alba. - J. Avian Biol. 39: 593-598.

Roulin, A., Dreiss, A. N., Fioravanti, C. and Bize, P. 2009. Vocal sib-sib interactions: how siblings adjust signalling level to each other. - Anim. Behav. 77: 717-725.

Roulin, A., da Silva, A. and Ruppli, C. A. 2012. Dominant nestlings displaying female-like melanin coloration behave altruistically in the barn owl. - Anim. Behav. 84: 1229-1236.

Ruppli, C. A., Almasi, B., Dreiss, A. N., Battesti, M., Jenni, L. and Roulin, A. 2012. Corticosterone promotes scramble competition over sibling negotiation in barn owl nestlings (Tyto alba). - Evol. Biol. 39: 348-358.

Schaeffel, F. and Wagner, H. 1996. Emmetropization and optical development of the eye of the barn owl (Tyto alba). - J. Compar. Physiol. 178: 491-498.

Steinbach, M. J. and Money, K. E. 1973. Eye movement of the owl. - Vision Res. 13: 889-891.

Teather, K. L. 1992. An experimental study of competition for food between male and female nestlings of the red winged blackbird. - Behav. Ecol. Sociobiol. 31: 81-87.

Treves, A. 2000. Theory and method in studies of vigilance and aggregation. - Anim. Behav. 60: 711-722.

Veiga, J. A., Roselino, E. S. and Miglirini, R. H. 1978. Fasting, adrenalectomy and gluconeogenesis in the chicken and a carnivorous bird. - Am. J. Physiol. 234: R115-121. 